\title{
Improving Postpartum Care: Identifying Opportunities to Reduce Postpartum Emergency Room Visits Among Publicly-Insured Women of Color
}

\author{
Taylor Harrell ${ }^{1}$ (1) Elizabeth A. Howell ${ }^{2}$ Amy Balbierz ${ }^{3,4,5} \cdot$ Luz Guel $^{6} \cdot$ Juan Pena ${ }^{7}$ Teresa Janevic ${ }^{3,4,7}$. \\ Ksenia Gorbenko ${ }^{3,8}$
}

Accepted: 12 October 2021 / Published online: 4 January 2022

(c) The Author(s), under exclusive licence to Springer Science+Business Media, LLC, part of Springer Nature 2021

\begin{abstract}
Background/Objectives The purpose of this study was to explore the postpartum experiences of publicly-insured women of color, and identify how postpartum care can be improved to reduce hospital emergency department usage after delivery. Methods We conducted four focus groups with 18 publicly-insured women who primarily self-identified as Black and/or Latina and gave birth between June 1, 2019 and May 1,2020. We used inductive qualitative analysis to identify prominent themes from focus group discussions.

Results We identified four domains: (1) lack of access to and communication with a medical team; (2) lack of preparation; (3) value of social support; and (4) participant-identified opportunities for improvement.

Conclusions for Practice This study describes the postpartum experiences of publicly-insured women of color with the objective of identifying areas for intervention to reduce postpartum emergency department usage. Our findings suggest that focused efforts on enhancing continuity of care to increase healthcare access, strengthening patient-provider communication by training providers to recognize unconscious bias, increasing postpartum preparation by adapting teaching materials to an online format, and engaging women's caregivers throughout the pregnancy course to bolster social support, may be beneficial.
\end{abstract}

Keywords Maternal health $\cdot$ Postpartum $\cdot$ Emergency department $\cdot$ Health disparities $\cdot$ Medicaid-insured

\section{Significance}

Clinical domains other than obstetrics have recognized the importance of care management interventions to reduce hospital emergency department visits. While recent literature

Taylor Harrell

taylor.harrell@icahn.mssm.edu

1 Department of Medical Education, Icahn School of Medicine at Mount Sinai, One Gustave L. Levy Place, New York, NY 10029, USA

2 Department of Obstetrics and Gynecology, Perelman School of Medicine, University of Pennsylvania, Philadelphia, PA 19104, USA

3 Department of Population Health Science and Policy, Icahn School of Medicine at Mount Sinai, One Gustave L. Levy Place, Box 1077, New York, NY 10029, USA

4 Blavatnik Family Women's Health Research Institute, Icahn School of Medicine at Mount Sinai, New York, NY 10029, USA has identified clinical predictors of postpartum ED visits, interventions to reduce unnecessary ED visits and improve postpartum health for high-risk women are limited. This study reveals opportunities for early intervention to reduce the widening gap of maternal morbidity and mortality for

5 Grossman School of Medicine, New York University, New York, NY 10016, USA

6 Department of Environmental Medicine \& Public Health, The Mount Sinai Transdisciplinary Center on Early Environmental Exposures, Icahn School of Medicine at Mount Sinai, 17 East 102nd Street, New York, NY 10029, USA

7 Department of Obstetrics, Gynecology, and Reproductive Science, Icahn School of Medicine at Mount Sinai, One Gustave L. Levy Place, Box 1070, New York, NY 10029, USA

8 Health Care Delivery Science, Mount Sinai Health System, New York, NY 10029, USA 
low-income women of color and to improve their postpartum experience.

\section{Introduction}

Racial and ethnic disparities in maternal health are a public health crisis in the United States. Black women are two to three times more likely to experience severe maternal morbidity and three to four times more likely to suffer pregnancy-related mortality compared to non-Hispanic white women (Callaghan, 2012). These elevated health risks exist across the pregnancy continuum and extend into the postpartum period. Black women are more likely to have an emergency department (ED) visit after delivery (Ehrenthal et al., 2017), and Black and Latina women have a two-fold increased risk of postpartum hospital readmission relative to white women (Aseltine et al., 2015). Postpartum ED visits and hospital readmissions are markers of the excess morbidity Black and Latina women experience and studies indicate that some instances of emergency service utilization following delivery are preventable (Brousseau et al., 2018).

The postpartum period presents a critical opportunity to improve outcomes (Clapp et al., 2016), given that $51 \%$ of maternal deaths occur within 1 day to 1 year after delivery (Petersen et al., 2019). Recent value-based payment models have focused on moving care upstream and centering intervention on providing incentives to increase adherence to the 6-week postpartum visit. However, these interventions may have missed the mark as many women experience health problems long before the 6-week time point, resulting in increased emergency department visits (Howell et al., 2017). In fact, studies indicate that $60 \%$ of all maternal ED visits occur prior to 6 weeks postpartum (Harris et al., 2015). Understanding trends in postpartum ED utilization is central to addressing disparities as a marker of poor maternal health, future morbidity, and pregnancy's long-term impact on the health and wellbeing of new mothers and their families.

Clinical risk factors (e.g. comorbidities, delivery type, psychiatric illness) for postpartum complications and ED usage have been extensively described (Clark et al., 2010; Ehrenthal et al., 2017; Harris et al., 2015). Yet, the current body of literature lacks the patient perspective on what brings women to the ER, and whether opportunities for intervention existed along their journey.

Over the last decade, research has focused on understanding the role of social determinants of health (SDoH) - the conditions in which people are born, grow, live, work, and age-in relationship to health outcomes (Susser, 2004). However, increasing evidence argues that addressing $\mathrm{SDoH}$ alone does not completely improve outcomes for women of color because it fails to recognize the larger systemic context that serves as the primary driver of social inequity (Martin \& Montagne, 2017; New York City Department of Health and Mental Hygiene, 2016). Newer approaches suggest investigating the structural determinants of health, defined as the cultural norms, policies, institutions, and practices that define the distribution (or maldistribution) of $\mathrm{SDoH}$, as the root cause of health disparities and a primary driver of SDoH (Braveman, 2014; Crear-Perry et al., 2021; Yearby, 2020). The historical allocation of greater resources to white communities while disproportionately depriving communities of color is an important lens to consider in maternal morbidity and mortality outcomes because it allows us to understand how racism, classism, and sexism intersect to affect the lives of women of color.

Identifying key factors that lead to increased rates of postpartum ED visits can inform quality improvement efforts. However, a more thorough investigation into the patient lived experiences driving ED visits, especially for disadvantaged women of color, is needed. The objective of this study is to explore the postpartum experiences of publicly-insured women of color to (1) identify additional risk factors that may contribute to postpartum emergency room usage and (2) identify potential opportunities for intervention.

\section{Methods}

We used purposive sampling to recruit postpartum women into four focus groups in the summer and fall of 2020. All women were eligible to participate if they spoke Spanish or English, $\geq 18$ years old, delivered an infant between June 1, 2019 and May 1, 2020 at Mount Sinai Hospital, and had Medicaid coverage for their delivery. Eligible participants were identified using either an EPIC query or outreach to the Ambulatory Clinic by posting IRB approved recruitment fliers in the waiting room. All participants verbally gave their informed consent by phone with use of a witness prior to inclusion in the study and received a copy of the consent form via mail or email. Participants also completed a brief anonymous demographic survey via email and mail. Participants were offered a \$100 gift card and round-trip MetroCard for compensation of their time upon completion of the focus group. Race/ethnicity-concordant moderators $(\mathrm{TH}$, LG, JP) led the focus groups. Three English focus groups $(\mathrm{N}=12)$ were conducted in July and August 2020 and one Spanish focus group $(\mathrm{N}=6)$ was conducted in October 2020. Due to COVID-19 health and safety concerns, the focus groups were held virtually using a HIPAA compliant platform. Approval of this study was obtained by the Program for the Protection of Human Subjects at the Icahn School of 
Table 1 Moderator guide

\begin{tabular}{|c|c|}
\hline Construct & Question examples \\
\hline Warm-up & $\begin{array}{l}\text { Think back to your first few days after delivering your baby } \\
\text { 1. If you could paint a picture of yourself and how you were feeling, what sticks out the most? } \\
\text { 2. Were there any major problems you encountered in the first weeks after your delivery? }\end{array}$ \\
\hline Psychological well-being & $\begin{array}{l}\text { 1. How were you feeling emotionally after coming home from the hospital? } \\
\text { 2. Did you feel prepared to deal with any difficulties you faced? }\end{array}$ \\
\hline Social support & $\begin{array}{l}\text { 1. What kind of supports do you have at home? } \\
\text { 2. In what ways are family/friends helpful? In what ways were they not helpful? }\end{array}$ \\
\hline Healing after childbirth & $\begin{array}{l}\text { 1. Did you have any major health issues after you had your child? Please tell us about those issues } \\
\text { 2. Did any of you go to the Emergency Room for care during this period for your own health? If yes, please let us } \\
\text { know why }\end{array}$ \\
\hline Barriers to care & $\begin{array}{l}\text { 1. If you had to visit your doctor prior to your scheduled postpartum visit, how easy was it to schedule an appoint- } \\
\text { ment able to see your ob/midwife? } \\
\text { 2. Was there anything that prevented you from receiving care when you first had symptoms? }\end{array}$ \\
\hline Patient education & $\begin{array}{l}\text { 1. What key things did your doctor or midwife do that helped you understand and take care of yourself physically and } \\
\text { emotionally? }\end{array}$ \\
\hline Wrap up & $\begin{array}{l}\text { 1. If you were going to make one recommendation to a new mom to help her during the first months postpartum what } \\
\text { would that be? }\end{array}$ \\
\hline
\end{tabular}

Medicine at Mount Sinai School. This study was conducted in accordance with the COREQ criteria for reporting qualitative research (Tong et al., 2007).

A moderator guide containing open-ended questions (Table 1) was used by the moderators to ensure consistency among focus groups and techniques such as reflection (e.g. "Let me repeat what you said") were used to clarify statements. The moderator guide was developed by the research team based on prior research and published literature. The same questions were asked in each focus group and were only slightly modified to follow the natural flow of the conversation. Each focus group lasted approximately an hour in length. The focus groups were both video and audio-recorded and transcribed in their entirety. The Spanish focus group transcript was professionally translated into English and bilingual members of the research team verified the accuracy of the translation. Following the focus groups, the research team debriefed about initial thoughts and impressions. The moderator then wrote detailed notes summarizing each focus group, including verbatim quotes from the transcripts. We used a combined inductive and deductive approach to analyze the data. First, we developed deductive codes based on the team's impressions from the focus groups and the moderator guide topics. Iterative review of the transcripts and coder triangulation were used to thematically analyze the data. Members of the research team individually reviewed the transcripts and then met to create an inclusive master themes list. Two independent readers then coded the transcripts and a third reader reviewed the coded transcripts for inter-rater agreement. Disagreements were then discussed and resolved. Dedoose software was also used to facilitate data management and retrieval (Dedoose Version 8.0.35, 2018).

\section{Results}

Eighteen women participated in one of four focus groups (Table 2). Majority of participants self-identified as Black/African-American and were over the age of 30. Of eighteen participants, five women had an ER visit within 30 days of delivery. Reasons for ER visits included preeclampsia, postpartum cardiomyopathy, and infection.

Four major themes and 13 sub-themes were identified that described women's postpartum experiences (Table 3). The four major themes included: (1) lack of access to and communication with a medical team, (2) lack of preparation, (3) importance of social support and (4) participant recommendations for improving quality of care. Each theme is discussed below. Tables 4, 5, 6, and 7 include the themes and representative quotes from participants.

\section{Lack of Access to \& Communication with Medical Team}

Participants reported varying degrees of accessibility and communication with their medical team following delivery. Some women reported no problems with scheduling appointments. Other participants, particularly women who had postpartum complications, identified hardships 
Table 2 Demographic characteristics of the study participants $(\mathrm{N}=18)$

\begin{tabular}{|c|c|}
\hline Participant characteristics & $\begin{array}{l}\text { Par- } \\
\text { ticipants } \\
(n=18)\end{array}$ \\
\hline \multicolumn{2}{|l|}{ Age } \\
\hline Below 30 & 5 \\
\hline $30+$ & 10 \\
\hline \multicolumn{2}{|l|}{ Primary language } \\
\hline English & 8 \\
\hline Spanish & 4 \\
\hline Other & 3 \\
\hline \multicolumn{2}{|l|}{ Race } \\
\hline Black or African American & 10 \\
\hline Asian & 1 \\
\hline White & 1 \\
\hline Declined to Answer & 3 \\
\hline U.S. origin (Y) & 8 \\
\hline Hispanic (Y) & 7 \\
\hline Married/living with partner (Y) & 6 \\
\hline \multicolumn{2}{|l|}{ Highest education level } \\
\hline High school graduate or GED & 4 \\
\hline Some college or above & 11 \\
\hline First pregnancy (Y) & 7 \\
\hline \multicolumn{2}{|l|}{ Parity } \\
\hline One & 7 \\
\hline More than one & 8 \\
\hline Employed (Y) & 8 \\
\hline \multicolumn{2}{|l|}{ Annual income } \\
\hline Less than $\$ 45,000$ & 6 \\
\hline More than $\$ 45,000$ & 2 \\
\hline I don’t remember/don't know & 7 \\
\hline ER visits (Y) & 5 \\
\hline
\end{tabular}

Based on 15 responses. Three missing information

in accessing prompt care following delivery. Many women described feeling like a "bother" or "pain in the butt" to their doctor when calling to ask questions. As one woman explained:

It's hard getting in contact with someone when you're going through something. Sometimes you call and they tell you, "we are going to send the message and the doctor's going to call you back within $\mathrm{X}$ amount of days. But sometimes they don't call you back and it's like I have this thing going on, I'd rather be seen. [FG2, participant with ED visit]

Participants also spoke about their concern with not having a single team to watch over them prenatally, during delivery, and after birth. Due to being Medicaid-insured, most participants had rotating providers throughout their pregnancy course and postpartum. For one participant who experienced an adverse health event, inability to identify a single provider made the emergency room seem like the next-best option to receive the immediate care she needed. Women also described having rotating providers as a barrier to building trusting relationships with their physician. Participants described being "a part of [a] huge system" that prevented development of emotional connections to their doctors, and instead relying on visiting nurses and midwives for support.

Additionally, some women reported racism and bias in their interactions with healthcare providers throughout prenatal care and during delivery, which dampened their communication and desire to reach out to them. Prompts about racism and bias sparked a robust conversation in the Spanish-speaking focus group, with one woman remarking:

She [a doctor] told me you should learn English... [or] you're going to live in this world and you won't understand anything. [FG4]

When asked directly about racism and bias, some participants described providers as "very kind" and the services they received as "excellent." Others emphasized the structural barriers they experienced due to being low-income and Medicaid-insured, such as lengthy clinic wait times and financial disadvantages, that made it difficult to access care. One participant compared her experience being Medicaidinsured with being privately insured, stating:

The first time I gave birth, I had insurance through work...But I lost my insurance and this time I delivered with Medicaid...I never felt like I had a team of people watching. Even while I had the gestational diabetes, it was a total experience from the last time because I had a specific doctor that only looked at me. [FG2, participant with ED visit]

\section{Lack of Preparation for Postpartum Period}

A general response among all focus groups was feeling unprepared for what to expect following delivery. Some participants specifically emphasized being ill-prepared for the emotional aspect, while others spoke about underestimating the lifestyle changes of bringing home a new child. One participant stated, "nothing can really prepare you for what is about to go down" [FG3, participant without ED visit]. Participants who had experienced a postpartum complication tended to recall the fear they felt during the postpartum period and beyond by using words such as "terrifying" and "scary" when recounting the post-delivery experiences with which they felt unequipped to deal.

Women stated that they did not receive enough health education about what to expect or look out for during the postpartum period, which contributed to feelings of 
Table 3 List of focus group themes and subthemes

Themes and sub-themes

1. Lack of access to and communication with medical team

1.1 Rotating providers

1.2 Trouble building trusting relationships

1.3 Racism \& bias

2. Lack of preparation for postpartum period

2.1 Fear

2.2 Health education

2.3 Difficulty retaining information

3. Importance of social support to postpartum success

3.1 Identifying a close support person

3.2 Role of partner

3.3 Unmet expectations

4. Participant recommendations for improving quality of care

4.1 Enhancing health education

4.2 Engaging caregivers

4.3 Support groups

4.4 Listen to women

Table 4 Lack of access to and communication with medical team

Sub-theme 1.1: Rotating providers

i. The truth is I was attended more or less by five or six midwives. It would have been nice to have just one doctor until the end for delivery

ii. Maybe if we had one doctor, it would be easier for us to contact that one doctor, communication would be better for us and treatment could

be sooner because that doctor knows about our health conditions and what's going on with us

iii. Everyone that I had met through the clinic... were really great and helpful and I liked them but I never felt like I had a team of people watching. I don't know who I could've called in an emergency. I didn't feel that I had a specific doctor to reach out to to ask questions. So my reaction was to just go to the emergency room instead of calling the clinic first

Subtheme 1.2: Barriers to building trusting relationships

i. I only saw her [the doctor] at the postpartum meeting where she told me she felt really bad for me that I went through this. But it felt really detached [because] she wasn't really a part of what happened to me

ii. Because it's so busy everyone is all over the place, it's hard. As [a] woman you may not feel comfortable...how can I open up and tell you things? You're all over the place

iii. It was always my midwife who attended me. Even when I was in the hospital...she went to see me [on] the floor where I stayed for a month. Every morning she would go to see me, to check everything [and] how I was

Subtheme 1.3: Racism \& bias

i. [A doctor] looked at me, 'do you speak English?' That was very upsetting for me. I wasn't sure if he meant it in a really racist way, but I was shocked. It was very awkward and I didn't want to speak to that man after that

ii. I feel like a lot of women don't get services because of financial reasons or don't have the insurance that covers those types of things. Services should be offered to women and let them decide if they would like to decline

iii. I contacted the doctors so much in the hospital that someone spoke to the social worker, and said I should see someone else, like a psychiatrist or something

unpreparedness. Although participants recognized that they received some education from providers, they often felt like this information was insufficient. One participant noted that she was predominantly, "relying on [herself] and getting information online" while another participant referred to Google as being her "best friend." As one woman shared:
I felt like I [was] in the dark, this is my first baby. [FG1, participant without ED visit]

Women, both who did and did not have an ED visit, expressed difficulties in retaining information immediately following delivery. Women repeatedly described it as a "blur" and therefore advised against verbal education during clinic or hospital visits as the main educational tool. 
Table 5 Lack of preparation for postpartum period

Subtheme 2.1: Fear

i. This was the first time out of all of my pregnancies that I ever experienced preeclampsia or any other type of complication. I usually had a normal pregnancy, no complications at all. So this was a very scary experience for me

ii. When it happened it felt like a death sentence and it was quite confusing and scary because I had no idea that a pregnancy could lead to heart failure

Subtheme 2.2: Lack of education

i. It didn't feel like a big deal the way that they explained how I had to keep track of my blood pressure. I don't know, maybe they could have given me a little bit more information on issues that can come up from high blood pressure

ii. At my postpartum appointment she told me that $99 \%$ of women go through labor and come out healthy....and nothing happens, but that $1 \%$ was me. I really could have died. I felt very unprepared. I understood...the other high-risk issues that happen with older women having kids but a heart problem I never guessed. That should have been more discussed with me... it would have been easier for me to catch it

Subtheme 2.3: Difficulties in retaining information

i. I wasn't really listening to what she [the midwife] was saying, I just wanted to go home for me to have time with my daughter. She was talking, but it wasn't really sinking into my mind. I was like, 'can I go now?' so I wasn't really paying attention

ii. Right after giving birth it's all kind of a blur and you're trying to focus all of your energy on your child, and you have more children at home

Table 6 Importance of social support to postpartum success

Subtheme 3.1: Identifying a close support person

i. you... think you know everything but you don't know. So if you have someone that really wants to help you, talk to that person and see whatever help that they can give to you

ii. There's really not a book [on] how to be a parent. I feel like the support system is very very important in order to raise a child, have a child, and take care of the child. Even if you went to classes, it is very difficult. So have a good support system

Subtheme 3.2: Role of partners

i. [W] hen we came home, my husband was here... we were doing it together, like we were a team, he was helping a lot with the baby

ii. My husband also took me to the Emergency Room. It was just him who was with me, and he was in charge of rushing home to see my eldest children, and rushing back to the hospital and to be with me, just him

Subtheme 3.3: Unmet expectations

i. I live with everyone at home, my mom, my dad, and my siblings. And at that time, when I gave birth, I'd have liked some of my family to help me the day my child went to sleep, maybe I could go to sleep too... but for some reasons, they had to work, each of them had to do their things, they... didn't do it

ii. Certain expectations that I had when they didn't happen, that was really disappoint[ing]

Participants also spoke about competing demands at the time of delivery such as other children or wanting to return home from the hospital, that made it difficult to focus on the information that was being provided.

\section{Importance of Social Support to Postpartum Success}

The value of social support spanned all focus groups. Women either described feeling grateful for the support they received, or reported feeling overwhelmed because support was lacking. One participant spoke directly about the challenges of asking for help as a new mom, stating: "there is no longer a village" to help with your child [FG1, participant without ED visit].

Some participants stressed the importance of identifying a close support person during pregnancy to prepare for unexpected events in the postpartum period. Women discussed how their partners, mothers, mothers-in-law, sisters and older children helped them in the first few weeks or months after coming home from the hospital. Most participants spoke about the important role of partners in coping with new responsibilities during the postpartum period.

For women who needed to go to the ER, partners played an essential role as family caretakers. However, not everyone had a husband or a partner to help them. For example, one woman mentioned that she split from her partner right after the baby was born and another had a husband who lived in another country. Other women expressed disappointment of unmet expectations when family members were not supportive or able to help. 
Table 7 Participant recommendations for improving quality of care

Subtheme 4.1: Enhancing health education

i. ... maybe, even if it is a very severe case like the one $\mathrm{I}$ had, maybe if those things would have been listed, maybe my husband would have read it and kept it

ii. Pay attention to moms postpartum. Providing resources or information right before you give birth or while they are in the hospital, 'this is what you need to be careful of.' A list of things that you could use or that may use could be life changing. Critical for mom

iii. Come up with the right kind of resources, whether it's pamphlets, whether it is [an] online app

Subtheme 4.2: Engage caregivers

i. ... in case you are ill, the family already knows what you have, and you feel that support because the doctor... already told them that she needed that support on this, this and this. Or for example, when you have an appointment, that your relative doesn't stay outside but that they may enter with you and listen to your opinion

ii. it's a good initiative to do at least a kind of talk, in order to involve the family member at the beginning and near the end of the pregnancy, so that they can understand that there is a new member coming into the family, that is, that everyone must collaborate, that everyone must be supportive. Because, sometimes, as a new mother, as it is my case, I didn't know if I am doing well with the baby, or I am feeling very tired, or I need someone to tell me "I am going to stay with you tonight to watch your baby". I do think that family members should be more involved directly from the center itself, so that family members feel more engaged than if

I tell them myself."

Subtheme 4.3: Support groups

i. I felt like I had enough support at home but to be connected to other women who are actually going through it. Because to be honest it's hard for me to find anyone in New York that has a group like that. And it would've been nice I guess to be connected. With someone who has experienced it or is going through it right now

ii. So I did... my doctor visits with a whole bunch of other girls who were expecting around the same time. So, I was supposed to give birth in June. So, my doctor was really really available when it came to emailing and answering questions. If I didn't ask her, I'd ask my nurse. If it wasn't the nurse then I would look for the mommy, the mommy, the little groups that were of women pregnant at the same time as well to ask them questions

Subtheme 4.4: Listen to women

i. A doctor in the room would not listen to me. I kept telling him, I don't want forceps. He got frustrated

ii. One thing doctors can do is listen more. A lot of simple thing [s] but a lot of doctors are not doing it

\section{Participant Recommendations for Improving Quality of Care}

Women in each focus group were asked to provide direct feedback on how postpartum care could be improved. In this section, we provide a high-level summary of the identified opportunities for intervention that were consistent across focus groups.

Several of our participants mentioned that enhancing health education by providing adapted material formats for both themselves and to share with caregivers could be helpful, particularly in printed form to take home or an easily accessible electronic backup in case the printout gets lost. One participant expressed a desire for more health education about possible warning signs.

Women in our focus groups spoke about the need for health care providers to engage caregivers and family members from the start. Although partners and family members played an important support role for many of our participants, they were not always knowledgeable or helpful. According to one woman, “...maybe, even if it is a very severe case like the one I had, maybe if those things would have been listed, maybe my husband would have read it and kept it." [FG2, participant with ED visit]
Aside from family members, one participant mentioned seeking support from peers who experienced a similar postpartum complication. Another woman agreed that creating informal, peer support groups of women who are going through the same experience can be helpful and indicated that participating in a focus group gave her a different perspective. One participant described a positive experience with being part of a group of women treated in the same clinic who were expecting around the same time. However, the continuation of such connections into the postpartum period remains unclear.

In closing remarks, multiple participants added that providers should "just listen to women more."

\section{Discussion}

Our study identified the following factors that influenced women's postpartum experiences: access and communication with their medical team, perceived level of preparedness for the postpartum period, and social support network following delivery. While some women had good communication with their providers, many women reported barriers due to rotating providers and racism/bias. Generally, 
all women felt a level of unpreparedness for the postpartum period due to ineffective education methods. This lack of knowledge often resulted in fear for women who experienced warning signs prompting ED use. Social support networks were essential to postpartum satisfaction, and greatly influenced women's ability to juggle the changing responsibilities of bringing home a new baby.

Our results are consistent with previous studies that have found that primary care accessibility, health education, and social support are critical factors to decreasing emergency service utilization and preventing adverse health outcomes among vulnerable populations (Hastings et al., 2008; O'Malley, 2013; Patel et al., 2018). However, our study adds that addressing these factors is particularly important in reducing postpartum morbidity and mortality among publicly-insured women of color due to the baseline systemic inequities that exist as a consequence of racism, classism, and sexism. To our knowledge, this study is the first to draw directly on the narratives of Black and Latina women to identify non-clinical risk factors that may be important for postpartum ED utilization.

Although we expected to find notable psychosocial differences between women who visited the emergency department and women who did not, each group had comparable experiences and shared similar concerns. Instead, our results indicated that the upstream structural factors of their experience due to being low-income, publicly insured women of color disproportionately affected the women who developed health complications during the postpartum period. Many of the concerns including inaccessibility of care, low levels of health literacy, and insufficient support reflect the historical, systemic, structural, and political forces that have limited the ability of communities of color, and in this context specifically women of color, to lead healthy lives (Crear-Perry et al., 2021). As pointed out by Dr. Whitney Laster Pirtle in her paper on racial capitalism, the systems of racism and capitalism have disadvantaged racially minoritized and economically deprived groups for centuries and will continue to harm their lives even in the presence of interventions that focus on alleviating inequality at the individual and community level (Laster Pirtle, 2020). While our study highlighted possible opportunities for proximal intervention that will be discussed, we recognize that true eradication of the stark disparities in maternal health outcomes will require multiple additional interventions aimed at addressing the policies and practices that uphold structural racism and drive poorer outcomes for women of color.

Based on our findings, we provide the following recommendations for intervention to reduce morbidity and mortality for publicly-insured women of color during the postpartum period:
1. Improving continuity of care Extensive studies indicate that a regular and usual source of care can improve health outcomes, particularly for vulnerable populations (Politzer et al., 2001). Postpartum care is both essential for obstetric outcomes and can serve as a vital link between pregnancy and well-woman care. Policies and strategies to improve continuity of care should focus on team-based approaches that encourage coordination of services for women.

2. Incorporating early postpartum visits Prior studies have shown that women at increased risk for postpartum emergency department use may benefit from earlier scheduled postpartum visits (Batra et al., 2017). By providing earlier opportunities for care, our findings suggest that there may be an increased ability to catch serious complications early, before the patient needs to go to the ED.

3. Adapting teaching materials Due to high rates of ER use within 1-week of delivery, interventions focused on discharge planning and post-delivery education among obstetric patients may reduce ER visits. While recent studies have called for increasingly standardized discharge instructions to prevent unnecessary ED use (Brousseau et al., 2018), our findings suggests that educating women on warning signs/symptoms during the delivery hospital stay is not optimal. In addition to the standard provider-patient education, accessible short videos for postpartum women and their family members about what to expect in the postpartum period and the warning signs that should prod them to contact their medical team are critical to accessible patient education.

4. Using evidence-based methodology to reduce providers' implicit bias Addressing structural racism requires a multi-pronged approach from the systems-level down that is rooted in equity frameworks. However, some progress can be achieved with provider-level training on recognizing implicit biases and providing accountability structures that encourage providers to address racism. Examples of accountability structures include providing openly available standardized assessments that allow patients to document their treatment quality, which ultimately should be linked to models of care that are associated with payment.

This study had two main limitations. The first limitation is a small number of participants. Due to COVID-19, we altered our recruitment strategy and focus group setting from in-person to virtual. Consequently, we anticipated that we may experience difficulties meeting our recruitment targets due to factors such as varying rates of technology access and fluctuating availability of participants. However, during thematic analysis we concluded sufficient themes were saturated and provided rich information on 
which we were able to draw recommendations. Additionally, our study was conducted at a single, academic institution in an urban setting and should be further investigated in other contexts such as non-teaching and community health settings.

In summary, this study offers evidence for understanding postpartum ED visits as a metric that can be useful in assessing quality improvement. By identifying risk factors for postpartum ED visits and implementing early intervention, the excess burden of morbidity and mortality experienced by low-income, underserved women can be reduced. However, future interventions should focus on addressing the structural forces that shape the contextual experience and influence women of color's ability to lead healthy lives.

\section{Conclusion}

Our study provides an important perspective into the postpartum experiences of publicly-insured women of color that can be helpful in identifying areas for intervention to reduce the need for postpartum emergency department usage. Our focus groups elucidated that some risk factors are structural in nature and addressing the patient-level factors is not enough. Addressing some of these factors will require high-level policy interventions. However, some proximal interventions addressing structural elements of care delivery such as enhancing continuity of care to increase healthcare access, adapting teaching materials to improve preparedness and engage caregivers, and training providers to reduce implicit bias, may also be beneficial.

Acknowledgements Supported in part by Research Grant No. 25-FY20-12 from the March of Dimes Foundation and NIMHD R01MD016029. This work was also supported by the New York Academy of Medicine Margaret E. Mahoney Fellowship Award.

Author Contributions EH conceptualized, designed, and supervised the study. EH, TJ, AB, KG, and TH contributed to the implementation of the study. TH, JP, and LG facilitated focus groups. TH, KG, and AB developed code and completed data analysis/interpretation. Author TH wrote the manuscript with support from KG, AB, and TJ. All authors reviewed and approved the final version of the manuscript.

Funding This study was supported by the March of Dimes Grant No. 25-FY20-12 and NIMHD R01MD016029. The content is solely the responsibility of the authors and does not necessarily represent the official views of the March of Dimes or NIMHD.

Data Availability The data underlying this article are not publicly available to protect the privacy of study participants.

Code Availability The data that support the findings of this study are not publicly available due to them containing information that could compromise research participant privacy/consent.

\section{Declarations}

Conflict of interest The authors declare that they have no conflict of interest.

Consent to Participate Informed consent was obtained from all study participants.

Consent for Publication Obtained via the IRB approved consent form. Details that might disclose the identity of participants were omitted.

Ethical Approval All procedures performed involving human participants were approved by the Institutional Review Board of the Mount Sinai School of Medicine, in accordance with Mount Sinai's Federal Wide Assurances (FWA\#00005656, FWA\#00005651). The Department of Health and Human Services approved the human subject research [Initial request] from $2 / 24 / 20$ to $2 / 23 / 23$ inclusive.

\section{References}

Aseltine, R. H., Jr., Yan, J., Fleischman, S., Katz, M., \& DeFrancesco, M. (2015). Racial and ethnic disparities in hospital readmissions after delivery. Obstetrics and Gynecology, 126(5), 1040-1047. https://doi.org/10.1097/AOG.0000000000001090

Batra, P., Fridman, M., Leng, M., \& Gregory, K. D. (2017). Emergency department care in the postpartum period: California births, 2009-2011. Obstetrics and Gynecology, 130(5), 1073-1081.

Brousseau, E. C., Danilack, V., Cai, F., \& Matteson, K. A. (2018). Emergency department visits for postpartum complications. Journal of Women's Health, 27(3), 253-257.

Callaghan, W. M. (2012). Overview of maternal mortality in the United States. Seminars in Perinatology, 36(1), 2-6. https://doi.org/10. 1053/j.semperi.2011.09.002

Clapp, M. A., Little, S. E., Zheng, J., \& Robinson, J. N. (2016). A multi-state analysis of postpartum readmissions in the United States. American Journal of Obstetrics and Gynecology, 215(1), 113.e1-113.e10. https://doi.org/10.1016/j.ajog.2016.01.174

Clark, S. L., Belfort, M. A., Dildy, G. A., Englebright, J., Meints, L., Meyers, J. A., Frye, D. K., \& Perlin, J. A. (2010). Emergency department use during the postpartum period: Implications for current management of the puerperium. American Journal of Obstetrics and Gynecology, 203(1), 38.e1-38.e386. https://doi. org/10.1016/j.ajog.2010.02.033

Crear-Perry, J., Correa-de-Araujo, R., Lewis Johnson, T., McLemore, M. R., Neilson, E., \& Wallace, M. (2021). Social and structural determinants of health inequities in maternal health. Journal of Women's Health, 30(2), 230-235. https://doi.org/10.1089/jwh. 2020.8882

Dedoose Version 8.0.35. (2018). Web application for managing, analyzing, and presenting qualitative and mixed method data. SocioCultural Research Consultants. LLC Retrieved October 27, 2020 from https://www.dedoose.com/.

Ehrenthal, D. B., Gelinas, K., Paul, D. A., Agiro, A., Denemark, C., Brazen, A. J., III., Pollack, M., \& Hoffman, M. K. (2017). Postpartum emergency department visits and inpatient readmissions in a medicaid population of mothers. Journal of Women's Health, 26(9), 984-991. https://doi.org/10.1089/jwh.2016.6180

Harris, A., Chang, H. Y., Wang, L., Sylvia, M., Neale, D., Levine, D., $\&$ Bennett, W. (2015). Emergency room utilization after medically complicated pregnancies: A medicaid claims analysis. Journal of Women's Health, 24(9), 745-754. https://doi.org/10.1089/jwh. 2014.5125 
Hastings, S. N., George, L. K., Fillenbaum, G. G., Park, R. S., Burchett, B. M., \& Schmader, K. E. (2008). Does lack of social support lead to more ED visits for older adults? The American Journal of Emergency Medicine, 26(4), 454-461. https://doi.org/10.1016/j. ajem.2007.07.005

Howell, E. A., Padrón, N. A., Beane, S. J., Stone, J., Walther, V., Balbierz, A., Kumar, R., \& Pagán, J. A. (2017). Delivery and payment redesign to reduce disparities in high risk postpartum care. Maternal and Child Health Journal, 21(3), 432-438. https://doi. org/10.1007/s10995-016-2221-8

Laster Pirtle, W. N. (2020). Racial capitalism: A fundamental cause of novel coronavirus (COVID-19) pandemic inequities in the United States. Health Education \& Behavior: The Official Publication of the Society for Public Health Education, 47(4), 504-508. https:// doi.org/10.1177/1090198120922942

Martin N. \& Montagne, R. (2017). Nothing protects black women from dying in pregnancy and childbirth. ProPublica. Retrieved August 6, 2021 from https://www.propublica.org/article/nothing-protectsblack-women-from-dying-in-pregnancy-and-childbirth.

New York City Department of Health and Mental Hygiene. (2016). Severe maternal morbidity in New York City, 2008-2012. New York, NY.

O'Malley, A. S. (2013). After-hours access to primary care practices linked with lower emergency department use and less unmet medical need. Health Affairs (project Hope), 32(1), 175-183. https:// doi.org/10.1377/hlthaff.2012.0494

Patel, P. B., Vinson, D. R., Gardner, M. N., Wulf, D. A., Kipnis, P., Liu, V., \& Escobar, G. J. (2018). Impact of emergency physicianprovided patient education about alternative care venues. The American Journal of Managed Care, 24(5), 225-231.
Politzer, R. M., Yoon, J., Shi, L., Hughes, R. G., Regan, J., \& Gaston, M. H. (2001). Inequality in America: The contribution of health centers in reducing and eliminating disparities in access to care. Medical Care Research and Review, 58(2), 234-248. https://doi. org/10.1177/107755870105800205

Petersen, E. E., Davis, N. L., Goodman, D., et al. (2019). Vital signs: Pregnancy-related deaths, United States, 2011-2015, and strategies for prevention, 13 states, 2013-2017. MMWR. Morbidity and Mortality Weekly Report, 68(18), 423-429.

Susser, E. (2004). Eco-epidemiology: Thinking outside the black box. Epidemiology (cambridge, Mass.), 15(5), 519-528. https://doi. org/10.1097/01.ede.0000135911.42282.b4

Tong, A., Sainsbury, P., \& Craig, J. (2007). Consolidated criteria for reporting qualitative research (COREQ): A 32-item checklist for interviews and focus groups. International Journal for Quality in Health Care, 19(6), 349-357.

Yearby, R. (2020). Structural racism and health disparities: Reconfiguring the social determinants of health framework to include the root cause. The Journal of Law, Medicine \& Ethics: A Journal of the American Society of Law, Medicine \& Ethics, 48(3), 518-526.

Braveman P. (2014). What are health disparities and health equity? We need to be clear. Public Health Reports, 129 (Suppl 2), 5-8. https://doi.org/10.1177/00333549141291S203

Publisher's Note Springer Nature remains neutral with regard to jurisdictional claims in published maps and institutional affiliations. 